\title{
Accurate Nano-Mechanical Mapping of Collagen I Fibrils
}

\author{
Sam Baldwin ${ }^{1}$, Sam Veres ${ }^{2}$, Michael Lee ${ }^{2}$ and Laurent Kreplak ${ }^{1}$. \\ 1. Department of Physics and Atmospheric Science, Dalhousie University, Halifax, Canada \\ 2. School of Biomedical Engineering, Dalhousie University, Halifax, Canada
}

The need for a quantitative methodology for determining the mechanical properties of materials at the nanoscale spans multiple fields of study. Recently much attention has been given to Peak Force Quantitative Nanomechanical Mapping (QNM) as a method of fulfilling this need. Peak Force QNM is a dynamic AFM mode where the cantilever is oscillated at $1 \mathrm{kHz}$, well below its characteristic resonance frequency. Instead of controlling the amplitude or the frequency as in other dynamic AFM techniques, Peak Force QNM uses the maximum force applied to the surface during each cycle as a control parameter. This method is intended to provide quantitative nanomechanical mapping of a sample by capturing an array of force curves while scanning a sample [1]. These force curves are then analyzed to provide separate maps of the mechanical properties of the sample. It has been shown that the roughness of a sample yields lower elastic modulus values and higher adhesion values compared to a smooth sample [2]. In this paper we present a methodology to analyze QNM data for samples exhibiting sudden changes in topography. We demonstrate the capability of the method on collagen I fibrils extracted from rat tails.

Collagen I fibrils were obtained from the dissection of rat tail fascicles. Fibrils in physiological solution were absorbed to a glass-bottom petri dish and then rinsed repetitively with deionized water while maintaining constant hydration to minimize alterations in the mechanical properties of the fibrils [3]. Scanasyst fluid + cantilevers from Bruker with a typical spring constant of $0.5 \mathrm{~N} / \mathrm{m}$ were calibrated using the thermal tune methodology in air and their deflection sensitivity was obtained from force curves acquired on a fused silica surface submerged in deionized water. Collagen Fibrils were targeted optically using differential interference contrast (DIC) microscopy and imaged by Peak Force QNM with a $20 \mathrm{~nm}$ pixel resolution. Figure la shows the Peak Force image generated by scanning a collagen fibril with a set point of $10 \mathrm{nN}$. It is clearly seen from the cross-section in Figure $1 \mathrm{~b}$ that the applied peak force varies by $\pm 40 \%$ of the peak force set point across the fibril. Unfortunately, the NanoScope Analysis software provided by Bruker uses the percentage of the peak force as fit boundaries when calculating the elastic modulus map. In order to ensure that all the force curves were being fitted between two specific force boundaries the force curves associated with the collagen fibril were extracted and then sorted by peak force value into $1 \mathrm{nN}$ bins. Once sorted the force curves were fit using the sneddon model. Force boundaries of 0.2 and $2 \mathrm{nN}$ were used in order to only fit an indentation depth corresponding to $10 \%$ of the fibril height [4]. Figure 2 contains two elastic modulus histograms obtained from the extracted force curves. The left one represents all force curves with peak force values between $5 \mathrm{nN}$ and $13 \mathrm{nN}$, while the right one represents all the force curves with a peak force value between $9 \mathrm{nN}$ and $11 \mathrm{nN}$. It is evident that sorting the data by peak force provides a more accurate measurement of the fibrils elastic modulus. By comparing these results with the cross section in Figure 1 it is apparent that the force curves from $9 \mathrm{nN}-11 \mathrm{nN}$ peak force correspond to the apex of the collagen fibril. This sorting approach provides an automatic way of discarding force curves acquired on the sides of the fibrils where tip slippage is likely to occur. 
a)

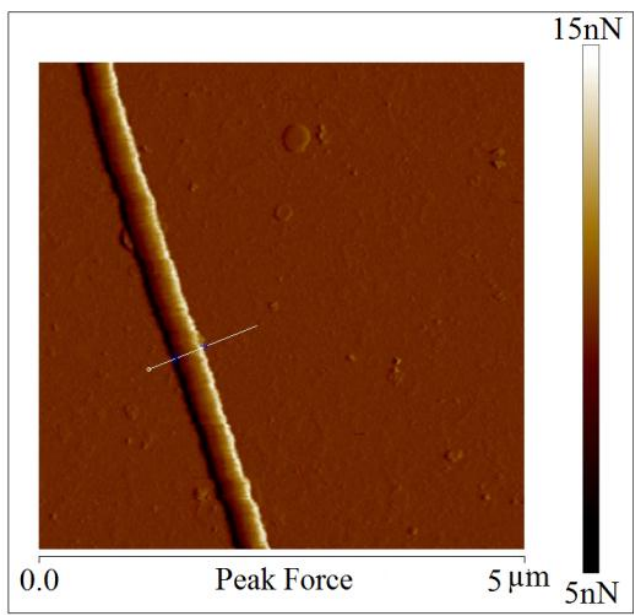

b)

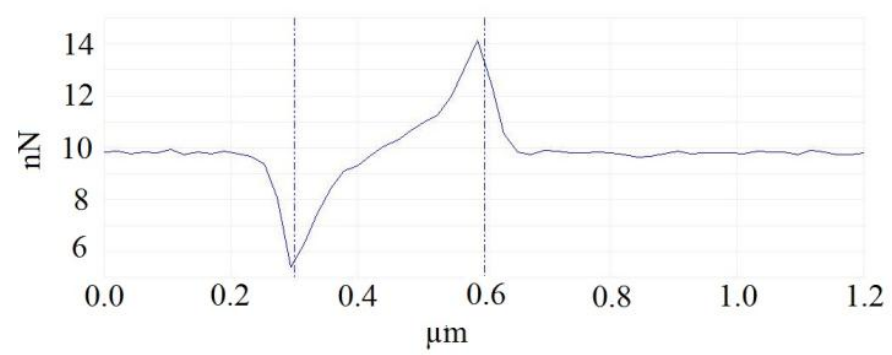

Figure 1. A demonstration of the variation in applied force across a collagen I fibril. a) a Peak force image of a collagen I fibril. b) A crossection of the collagen fibril peak force image showing the variance in applied force across the fibril.

a)

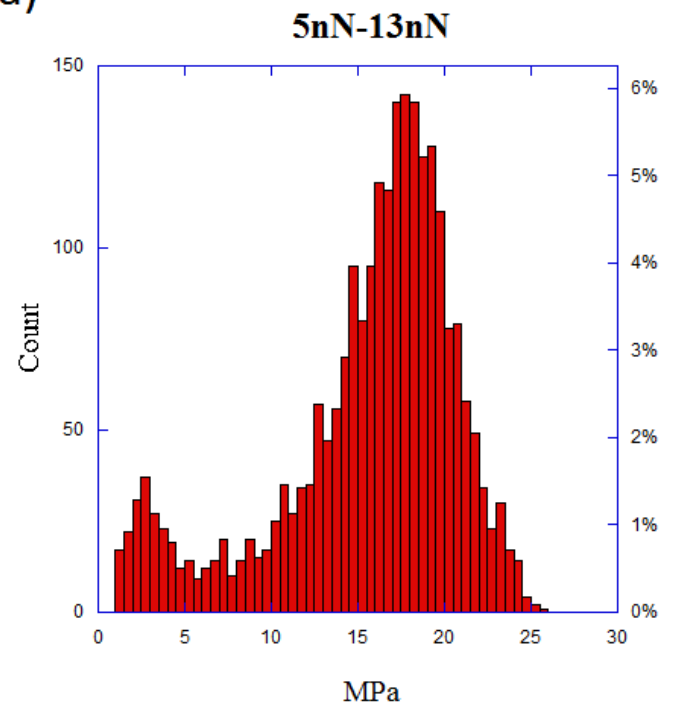

b)

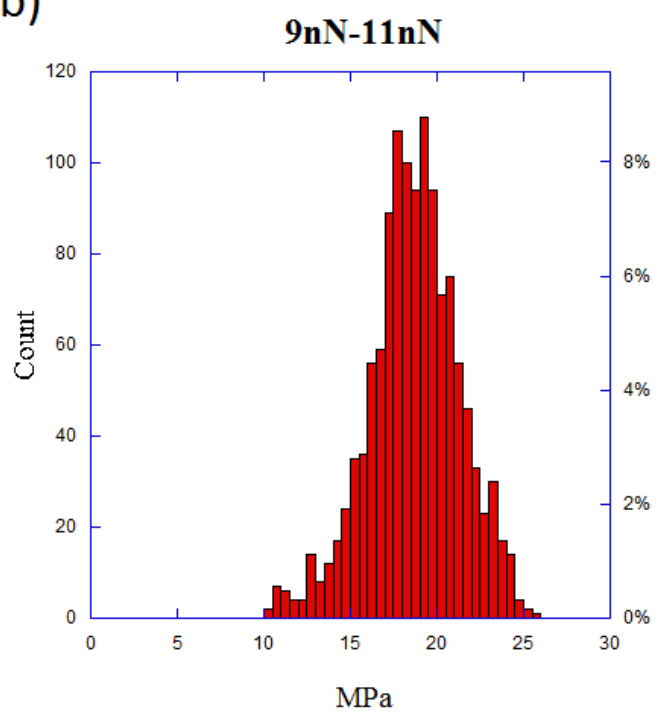

Figure 2. A comparison of the elastic modulus histograms from sorted extracted force curves from the collagen I fibril. a) All force curves with a peak force from $5 \mathrm{nN}-13 \mathrm{nN}$. b) All force curves with a peak force from $9 \mathrm{nN}-11 \mathrm{nN}$. The average mean values of the elastic modulus are $15.56 \pm 0.11 \mathrm{MPa}$ and $18.63 \pm 0.07 \mathrm{MPa}$ respectively.

\section{References:}

[1] Dokukin and Sokolov, Langmuir, 28 (2012), p. 16060

[2] P Trtik et al, Cement and Concrete Research 42 (2012), p. 215-221.

[3] M Wenger et al, Biophysical Journal 93 (2007), p. 1255-1263.

[4] H Bueckle in "The Science of Hardness Testing and Its Research Applications" American Society for Metals, (Materials Park), Ohio.

[5] The authors acknowledge funding from the Canada Foundation for Innovation, NSERC, and Dalhousie University. 